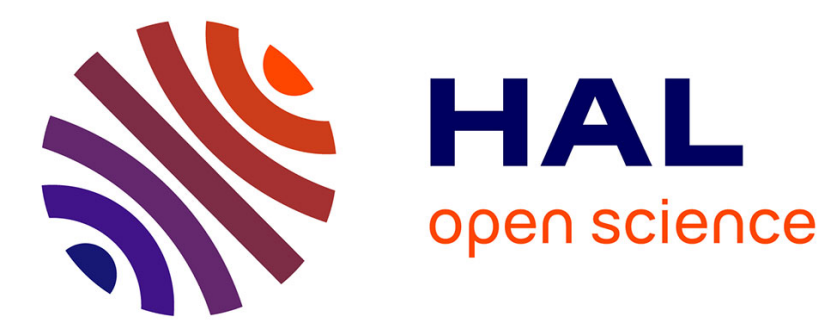

\title{
Improving the mass conservation of the level set method in a finite element context
}

Aymen Laadhari, Pierre Saramito, Chaouqi Misbah

\section{To cite this version:}

Aymen Laadhari, Pierre Saramito, Chaouqi Misbah. Improving the mass conservation of the level set method in a finite element context. Comptes Rendus. Mathématique, 2010, 348 (9-10), pp.535-540. 10.1016/j.crma.2010.03.011. hal-00444344v2

\section{HAL Id: hal-00444344 \\ https://hal.science/hal-00444344v2}

Submitted on 12 Mar 2010

HAL is a multi-disciplinary open access archive for the deposit and dissemination of scientific research documents, whether they are published or not. The documents may come from teaching and research institutions in France or abroad, or from public or private research centers.
L'archive ouverte pluridisciplinaire HAL, est destinée au dépôt et à la diffusion de documents scientifiques de niveau recherche, publiés ou non, émanant des établissements d'enseignement et de recherche français ou étrangers, des laboratoires publics ou privés. 


\title{
Improving the mass conservation of the level set method in a finite element context
}

\author{
Aymen LaAdhari, Pierre Saramito and Chaouqi Misbah
}

\begin{abstract}
In this paper, a new algorithm is proposed for improving the mass conservation of the level set method in the finite element context. Two kinds of Lagrange multipliers are introduced, associated respectively to the redistancing and advection equations. The first one, is located at the vicinity of the interface, while the second one is associated to a correction that is global to the domain. The performances of the proposed method are tested on the Zalesak test case, and the convergence rate versus the element mesh size are founded to be improved.
\end{abstract}

\section{Amélioration de la conservation de la masse pour la méthode des fonctions de niveaux en éléments finis}

\begin{abstract}
Résumé - Dans cet article, nous proposons un nouvel algorithme pour améliorer la conservation de la masse dans la méthode des fonctions de niveau dans un cadre éléments finis. Deux types de multiplicateurs de Lagrange sont introduits, associés respectivement à l'équation de redistanciation et à celle d'advection. Le premier est localisé au voisinage de l'interface, tandis que le second est associé à une correction globale au domaine de calcul. Les performances de la méthode proposée sont testées avec le cas test du disque de Zalesak, et nous observons que le taux de convergence par rapport au la taille des éléments du maillage est amélioré.
\end{abstract}

Keywords: level set method, mass conservation, finite element, Lagrange-Galerkin method

Version française résumée - Les méthodes numériques pour résoudre des problèmes à surface libres et des problèmes d'interfaces ont pris ces dernières années une importance croissante en physique et réalité virtuelle. Les applications recouvrent des domaines aussi variés que les interfaces liquide-vapeur, les mousses et les émulsions, les tsunamis ou le comportement d'un globule rouge dans une artère. La méthode des fonctions de niveau (voir par exemple $[3,6]$ ), s'appuyant sur un maillage fixe du milieu continu tridimensionnel, est devenue de plus en plus courante pour ce type de problème, car elle s'avère particulièrement souple lorsque les interfaces se déforment fortement ou présentent des changements topologiques (coalescence, etc). Une autre approche très répandue est la méthode de suivi lagrangien de l'interface par un maillage surfacique variable : moins apte aux changements topologiques, elle a cependant l'avantage de conserver mieux la masse, c'est à dire le volume du fluide, particulièrement pour des interfaces à courbure discontinue ou très minces. Des tentatives pour améliorer la conservation de la masse dans méthode des fonctions de niveau a a conduit à de nombreuses approches, telles que augmenter l'ordre des schémas [2]. Enright et al. [1], dans le cadre d'une méthode particulaire, a proposé d'utiliser des marqueurs lagrangiens pour reconstruire la fonction de niveau dans les régions sous-résolues. Une autre approche, proposée par Sussman et Fatemi [7] dans le cadre de la méthode des différences finies, consiste à ajouter une contrainte à l'équation de redistanciation discrète afin de mieux conserver la masse.

Dans ce papier, dans le cadre de la méthode des éléments fini, nous proposons deux améliorations indépendantes de la conservation de la masse discrète. La première amélioration concerne l'équation d'advection et introduit un multiplicateur de Lagrange scalaire, associé à la conservation globale de la masse. Ce multiplicateur peut être calculé explicitement et conduit à un coût négligeable en temps de calcul. La seconde amélioration est une extension aux éléments finis de la méthode proposée par Sussman et Fatemi : il s'agit d'introduire un multiplicateur de Lagrange local. Ce multiplicateur peut-être également explicité et est nul hors d'un voisinage de l'interface. La correction est ici également très peu coûteuse. Les performances de la méthode proposé sont testées avec le cas test du disque de Zalesak, et nous observons que le taux de convergence par rapport à la taille des éléments du maillage est amélioré.

Soit $\Lambda \subset \mathbb{R}^{N}$ un domaine borné, avec $N=2$ ou 3 , et $T>0$. Pour tout $\left.t \in\right] 0, T$, la surface fermée $\Gamma(t) \subset \Lambda$ s'écrit comme : $\Gamma(t)=\{(t, x) \in] 0, T[\times \Lambda ; \phi(t, x)=0\}$, c'est-à-dire comme le zéro d'une fonction 
de niveau $\phi(t,$.$) . Notons \Omega(t) \subset \Lambda$ la région où $\phi(t,$.$) est négatif et \Gamma(t)=\partial \Omega(t)$. Soit $\mathbf{u}$ la vitesse de $\Gamma(t)$ et

$$
\frac{D \phi}{D t}=\frac{\partial \phi}{\partial t}+\mathbf{u} \cdot \nabla \phi=0
$$

où $D \phi / D t$ désigne la dérivé matérielle. Le champs de vitesse $\mathbf{u}$ est supposé à divergence nulle. Cette équation de transport est complétée par une condition initiale $\phi(t=0)=\phi_{0}$, où, pour tout $x \in \Lambda$, la fonction $\phi_{0}(x)$ est une distance signée entre $x$ et la surface $\Gamma(0)$ :

$$
\phi_{0}(x)= \begin{cases}\inf \{|y-x| ; y \in \Gamma(0)\} & \text { si } x \notin \Omega(0), \\ \inf \{-|y-x| ; y \in \Gamma(0)\} & \text { sinon. }\end{cases}
$$

Cependant, le transport déforme la forme initiale de la fonction de niveau, qui n'est plus une distance signée pour $t>0$. Pour palier à cela, nous effectuons une ré-initilisation à une distance signée, appelée redistanciation, en calculant, à tout instant $t \in] 0, T$ [, la solution stationnaire du problème suivant, utilisant le pseudo-temps $\tau$ :

$$
\left\{\begin{aligned}
\frac{\partial d}{\partial \tau}(\tau, x ; t)+\operatorname{sgn}(\phi)(|\nabla d|-1) & =0 & & \text { p.p. }(\tau, x) \in] 0,+\infty[\times \Lambda, \\
d(0, x ; t) & =\phi(t, x) & & \text { p.p. } x \in \Lambda .
\end{aligned}\right.
$$

où $\operatorname{sgn}(\phi)$ est la fonction signe qui prend respectivement les valeurs $0,-1,+1$ sur l'interface $\Gamma(t)$, dans $\Gamma(t)$ et hors de $\Gamma(t)$. La solution stationnaire vérifie $|\nabla d|=1$ presque partout dans $\Lambda$, c'est-à-dire que $d(\infty, . ; t)$ est une distance signée, elle est prise comme la nouvelle fonction de niveau $\phi(t,$.$) à l'instant t$. Remarquons que la solution $d$ du problème de redistanciation (2) conserve la position de $\Gamma(t)$ : pour tout $\tau>0$, l'ensemble de niveau zéro de $d(\tau, . ; t)$ est égal à l'ensemble de niveau zéro de $\phi(t,$.$) . Il en résulte$ que le volume meas $(\Omega(t))$ est également conservé, ce qui est de première importance pour de nombreuses applications. Cependant, après discrétisation par différences finies ou éléments finis, cette propriété n'est satisfaite que de façon approchée.

Nous proposons un nouvel algorithme pour améliorer la conservation de la masse dans la méthode des fonctions de niveau dans un cadre éléments finis. Deux types de multiplicateurs de Lagrange sont introduits, associés respectivement à l'équation d'advection et à celle de redistanciation. Le premier est localisé au voisinage de l'interface, tandis que le second est associé à une correction globale au domaine de calcul. Les performances de la méthode proposée sont ensuite testées avec le cas test du disque de Zalesak, et nous observons que le taux de convergence par rapport à la taille des éléments du maillage est amélioré.

1. Maintaining the volume constraint. - Let us introduce the velocity field $\mathbf{v}=\operatorname{sgn}(\phi) \frac{\nabla d}{|\nabla d|}$. Then, problem (2) expresses equivalently as:

$$
\left\{\begin{aligned}
\frac{\partial d}{\partial \tau}(\tau, x ; t)+\mathbf{v} . \nabla d & =\operatorname{sgn}(\phi(t, x)) & & \text { a.e. }(\tau, x) \in] 0,+\infty[\times \Lambda, \\
d(0, x ; t) & =\phi(x, t) & & \text { a.e. } x \in \Lambda .
\end{aligned}\right.
$$

1.1. The volume constraint for the advection problem. - A new modification is introduced in the advection equation (1) related to the divergence free velocity field $\mathbf{u}$. At any time $t \in] 0, T[$, the global volume conservation writes:

$$
\frac{\mathrm{d}}{\mathrm{d} t}\left(\int_{\Omega(t)} \mathrm{d} x\right)=0
$$

A global Lagrange multiplier $\zeta(t) \in \mathbb{R}$ is introduced and the advection equation (1) becomes

$$
\frac{\partial \phi}{\partial t}+\mathbf{u} \cdot \nabla \phi+\zeta(t)=0
$$

The constraint (4) develops as:

$$
\frac{\mathrm{d}}{\mathrm{d} t}\left(\int_{\Omega(t)} \mathrm{d} x\right)=\frac{\mathrm{d}}{\mathrm{d} t}\left(\int_{\Lambda} H(\phi) \mathrm{d} x\right)=\int_{\Lambda} \delta(\phi) \frac{\partial \phi}{\partial t} \mathrm{~d} x=0
$$


Then, combining (5) and (6) leads to an explicit expression for $\zeta(t)$ :

$$
\zeta(t)=\frac{-\int_{\Lambda} \mathbf{u} \cdot \nabla \phi \delta(\phi) \mathrm{d} x}{\int_{\Lambda} \delta(\phi) \mathrm{d} x}
$$

Let us check that that since $\mathbf{u}$ is divergence free, then $\zeta$ is equal to zero and that the global volume correction will act at the discrete level only. For any integrable function $f$ on $\Gamma(t)$, let $\tilde{f}$ denotes an extension of $f$ in $\Lambda$. Then

$$
\int_{\Gamma(t)} f \mathrm{~d} s=\int_{\Lambda} \tilde{f} \delta(\phi)|\nabla \phi| \mathrm{d} x
$$

Let $\mathbf{n}=\frac{\nabla \phi}{|\nabla \phi|}$ denotes the unit outward normal to $\Gamma(t)$. By using (8) with $f=\mathbf{u . n}$ and then the Green formula:

$$
\int_{\Lambda} \mathbf{u} \cdot \nabla \phi \delta(\phi) \mathrm{d} x=\int_{\Gamma(t)} \mathbf{u} \cdot \mathbf{n} \mathrm{d} s=\int_{\Lambda} \operatorname{div} \mathbf{u} \mathrm{d} x
$$

1.2. The volume constraint for the redistancing problem. - Let us consider the redistancing problem at any fixed time $t>0$, while the pseudo-time $\tau$ is varying. Sussman and Fatemi [7] introduce the constraints that the volume remains constant in any $\mathcal{V} \subset \Omega(t)$ during the redistancing process:

$$
\frac{\partial}{\partial \tau}\left(\int_{\mathcal{V}} H(d) \mathrm{d} x\right)=0
$$

These authors introduces also a Lagrange multiplier $\lambda(\tau, x ; t)$ that enforces this constraint locally at $x \in \Lambda$. The evolution equation in (3) becomes:

$$
\left.\frac{\partial d}{\partial \tau}(\tau, x ; t)+\mathbf{v} . \nabla d=\operatorname{sgn}(\phi(t, x))+\lambda(\tau, x: t) g(d) \text { a.e. }(\tau, x) \in\right] 0,+\infty[\times \Lambda
$$

In order to ensure the volume conservation while not perturbing the redistancing process, the authors propose $g(d)=\delta(d)|\nabla d|$. Notice that (9) leads to

$$
\frac{\partial}{\partial \tau}\left(\int_{\mathcal{V}} H(d) \mathrm{d} x\right)=\int_{\mathcal{V}} \delta(d) \frac{\partial d}{\partial \tau} \mathrm{d} x=0
$$

Combining (10) and (11), Sussman and Fatemi [7] proposed to exploit numerically an explicit average value $\lambda_{\mathcal{V}}$ over an arbitrary finite volume $\mathcal{V} \subset \Lambda$ :

$$
\lambda_{\mathcal{V}}(\tau ; t)= \begin{cases}\frac{\int_{\mathcal{V}} \delta(d)(\mathbf{v} \cdot \nabla d-\operatorname{sgn}(\phi)) \mathrm{d} x}{\int_{\mathcal{V}} \delta(d) g(d) \mathrm{d} x} & \text { when } \mathcal{V} \cap \Gamma(t) \neq \emptyset \\ 0 & \text { otherwise }\end{cases}
$$

In the next section, based on the regularization procedure, a new local explicit expression of $\lambda(\tau, x ; t)$ can be exhibited and that $\lambda$ can be formally eliminated from the redistancing equation.

2. Regularization. - Three sharp functions have been introduced: the Heaviside function $H(\phi)$, that acts as the indicator of $\Lambda-\Omega$, the Dirac function $\delta(\phi)$ that localizes the interface $\Gamma$, and the sign function $\operatorname{sgn}(\phi)$. In order to avoid the discretization of the interface $\Gamma$, a transition region with a width of $2 \varepsilon$ is introduced and the Heaviside, the Dirac and the sign functions are replaced respectively by $H_{\varepsilon}(\phi), \delta_{\varepsilon}(\phi)$ and $\operatorname{sgn}_{\varepsilon}(\phi)$ as:

$$
\begin{aligned}
& H_{\varepsilon}(\phi)= \begin{cases}0, & \text { if } \phi<-\varepsilon \\
\frac{1}{2}\left(1+\frac{\phi}{\varepsilon}+\frac{\sin \left(\frac{\pi \phi}{\varepsilon}\right)}{\pi}\right), & \text { if }|\phi| \leq \varepsilon \\
1, & \text { otherwise }\end{cases} \\
& \delta_{\varepsilon}(\phi)=\frac{\mathrm{d} H_{\varepsilon}}{\mathrm{d} \phi}(\phi)= \begin{cases}\frac{1}{2 \varepsilon}\left(1+\cos \left(\frac{\pi \phi}{\varepsilon}\right)\right) & , \text { if }|\phi| \leq \varepsilon \\
0 & \text { otherwise }\end{cases} \\
& \operatorname{sgn}_{\varepsilon}(\phi)=2 H_{\varepsilon}(\phi)-1
\end{aligned}
$$


The regularization parameter is chosen as proportional to the the mesh size $h$.

After regularization the evolution equation (10) becomes:

$$
\left.\frac{\partial d}{\partial \tau}(\tau, x ; t)+\mathbf{v}_{\varepsilon} \cdot \nabla d=\operatorname{sgn}_{\varepsilon}(\phi(t, x))+\lambda(\tau, x ; t) f_{\varepsilon}(d) \text { a.e. }(\tau, x) \in\right] 0,+\infty[\times \Lambda
$$

where $\mathbf{v}_{\varepsilon}=\operatorname{sgn}_{\varepsilon}(\phi) \frac{\nabla d}{|\nabla d|}$ and $f_{\varepsilon}(d)=\delta_{\varepsilon}(d)|\nabla d|$. Conversely, the regularized version of the mass conservation (11) writes:

$$
\int_{\mathcal{V}} \delta_{\varepsilon}(d) \frac{\partial d}{\partial \tau} \mathrm{d} x=0, \quad \forall \mathcal{V} \subset \Lambda \quad \Longleftrightarrow \frac{\partial d}{\partial \tau}=0, \quad \text { when }|d|<\varepsilon
$$

Combining the two previous equations leads to a local version of the explicit expression for $\lambda$ :

$$
\lambda= \begin{cases}\frac{\operatorname{sgn}_{\varepsilon}(\phi)(|\nabla d|-1)}{\delta_{\varepsilon}(d)|\nabla d|} & \text { when }|d|<\varepsilon \\ 0 & \text { otherwise }\end{cases}
$$

Finally, the previous expression of $\lambda$ can be replaced in (16) and the evolution equation becomes, for any $(\tau, x) \in] 0,+\infty[\times \Lambda$ :

$$
\frac{\partial d}{\partial \tau}(\tau, x ; t)= \begin{cases}0 & \text { when }|\phi|<\varepsilon \\ -\mathbf{v}_{\varepsilon} \cdot \nabla d+\operatorname{sgn}_{\varepsilon}(\phi) & \text { otherwise }\end{cases}
$$

Notice that the set $\Gamma_{\varepsilon}(t)=\{x \in \Lambda ;|\phi(t, x)|<\varepsilon\}$ represents a thin layer around the boundary $\Gamma(t)$. Then, with the mass correction, the redistancing process acts only around a thin layer outside the boundary.

3. Time Discretization. - This section presents the numerical approximation of the advection problem (5) together with the redistancing problem (19), where the Lagrange multipliers are expressed by (7) and (12). Let $t^{n}=n \Delta t, n \geq 0$, where $\Delta t>0$ is the time step. Conversely, let $\tau^{m}=m \Delta \tau, m \geq 0$, where $\Delta \tau>0$ is the pseudo time step. Let $\phi^{n}, \zeta^{n}$ be approximations of $\phi(t), \zeta(t)$, respectively, at time $t^{n}$ and $d^{m}, \mathbf{v}^{m}$ be approximations of $d(\tau), \mathbf{v}(\tau)$ respectively at $\tau^{m}$. The time discretization is performed by using the method of characteristics: for any $t>0$ and $x \in \Lambda$, the characteristic curve $X(., x, ; t)$ passing at time $t$ through $x$ is defined by the following ordinary differential equation:

$$
\left\{\begin{aligned}
\frac{\partial X}{\partial t}(s, x ; t) & =\mathbf{u}(X(s, x ; t), t), \quad s>0 \\
X(t, x ; t) & =x
\end{aligned}\right.
$$

For any function $f(t, x)$, the total derivative $D f / D t$ expresses:

$$
\frac{D f}{D t}(t, x)=\left(\frac{\partial f}{\partial t}+\mathbf{u} . \nabla f\right)(t, x)=\left.\frac{\partial}{\partial t}\left(f\left(X\left(t, x ; t^{n+1}\right), t^{n+1}\right)\right)\right|_{\tau=t}
$$

Following Pironneau [4], this derivative is approximated by a first-order backward Euler scheme:

$$
\frac{D f}{D t}\left(t^{n+1}, x\right) \approx \frac{f\left(t^{n+1}, x\right)-f\left(t^{n}, X^{n}(x)\right)}{\Delta t}
$$

where $X^{n}(x)=x-\Delta t \mathbf{u}^{n}(x)$ denotes the first-order forward Euler approximation of $X\left(t^{n}, x ; t^{n+1}\right)$. The $n$-th step of the discrete scheme for the transport problem (5) becomes an explicit computation of $\phi^{n+1}$, where $\phi^{n}$ is known:

$$
\phi^{n+1}(x)=\phi^{n} \circ X_{\mathbf{u}}^{n}+\Delta t \zeta^{n}
$$

Conversely, the redistancing problem (19) is solved also explicitly:

$$
d^{m+1}= \begin{cases}d^{m} & \text { when }\left|\phi^{n}\right|<\varepsilon \\ d^{m} \circ X_{\mathbf{v}_{\varepsilon}}^{m}+\Delta \tau \operatorname{sgn}_{\varepsilon}\left(\phi^{n}\right) & \text { otherwise }\end{cases}
$$


Here, the characteristic have subscripts $\mathbf{u}$ and $\mathbf{v}_{\varepsilon}$ in order to avoid confusion. These algorithms are implemented in the C++ finite element library Rheolef [5], where the localization of $X^{n}(x)$ in an unstructured mesh is performed by using a fast searching algorithm based on a quad-tree data structure.

4. Space Discretization. - Let $\mathcal{T}_{h}$ be the finite element triangulation of $\Lambda$, and $V_{h}$ the space of continuous and piecewise linear functions on $\mathcal{T}_{h}$, where $h>0$ denotes as usual the mesh size parameter. Conversely, let $W_{h}$ be the space of piecewise constant functions on $\mathcal{T}_{h}$. At any step $n \geq 0$, the discrete version of the algorithm leads to a succession of explicit computations:

$$
\begin{aligned}
\tilde{\phi}_{h}^{n+1} & =\pi_{h}\left(\phi_{h}^{n} \circ X_{\mathbf{u}}^{n}\right) \\
\zeta_{h}^{n+1} & =\frac{-\int_{\Lambda} \mathbf{u} \cdot \nabla \tilde{\phi}_{h}^{n+1} \delta_{\varepsilon}\left(\tilde{\phi}_{h}^{n+1}\right) \mathrm{d} x}{\int_{\Lambda} \delta_{\varepsilon}\left(\tilde{\phi}_{h}^{n+1}\right) \mathrm{d} x} \\
\hat{\phi}_{h}^{n+1} & =\tilde{\phi}_{h}^{n+1}+\Delta t \zeta_{h}^{n}
\end{aligned}
$$

where $\pi_{h}$ denotes the Lagrange interpolation in $V_{h}$. Then, $\phi_{h}^{n+1}$ is obtained from $\hat{\phi}_{h}^{n+1}$ by the following discrete version of the redistancing algorithm. Let $d_{h}^{0}=\hat{\phi}_{h}^{n+1}$. At any step $m \geq 0$ of the redistancing algorithm, suppose $d_{h}^{m} \in V_{h}$ being known, and let $\mathbf{g}_{h}^{m} \in V_{h}^{N}$ be the approximation of $\nabla d_{h}^{m} \in W_{h}^{N}$ defined by the following linear system:

$$
\int_{\Lambda} \mathbf{g}_{h}^{m} \cdot \boldsymbol{\psi}_{h} \mathrm{~d} x=\int_{\Lambda} \nabla d_{h}^{m} \cdot \boldsymbol{\psi}_{h} \mathrm{~d} x, \quad \forall \boldsymbol{\psi}_{h} \in V_{h}^{N}
$$

A mass lumping procedure is used for this linear system: the integrals involved in the computation of the coefficients of the matrix associated to the $L^{2}$ scalar product are evaluated by using the trapeze quadrature formulae. By this way, the matrix of the linear system is replaced by a diagonal one, and the computation of $\mathbf{g}_{h}^{m}$ becomes explicit. Then, let

$$
\mathbf{v}_{\varepsilon, h}^{m}=\pi_{h}\left(\operatorname{sgn}_{\varepsilon}\left(\hat{\phi}_{h}^{n+1}\right) \frac{\mathbf{g}_{h}^{m}}{\left|\mathbf{g}_{h}^{m}\right|}\right)
$$

The discrete version of the redistancing algorithm writes also explicitly:

$$
d_{h}^{m+1}= \begin{cases}d_{h}^{m} & \text { when }\left|\phi_{h}^{n}\right|<\varepsilon \\ \pi_{h}\left\{d_{h}^{m} \circ X_{\mathbf{v}_{\varepsilon}}^{m}+\Delta \tau \operatorname{sgn}_{\varepsilon}\left(\phi_{h}^{n}\right)\left(1-\left|\nabla d_{h}^{m}\right|\right)\right\} & \text { otherwise }\end{cases}
$$

5. Numerical EXPERIMENTS. - The improvement of the accuracy with the mass conservation schemes is demonstrated for a classical test case: the rigid body rotation of Zalesak's disk in a constant rotating velocity field [8]: $\mathbf{u}(x, y)=(1 / 2-y, x-1 / 2)$ in the domain $\Lambda=] 0,1\left[^{2}\right.$. The initial data $\phi_{0}$ is associated to a slotted circle centered at $(1 / 2,7 / 10)$ with a radius $r=1 / 5$, the slope depth is $3 / 10$ and the width is equal to $1 / 10$. The triangular mesh is based on a $N_{h} \times N_{h}$ grid, where each square is subdivided in two triangles. The mesh size is $h=\sqrt{2} / N_{h}$ and $\Delta t=2 \pi / N_{h}$ is chosen. Fig. 1.b plots the error $\left\|H_{\varepsilon}(\phi)-H_{\varepsilon}\left(\phi_{h}\right)\right\|_{0,2, \Lambda}$ after $N_{h}$ time steps, at $t=2 \pi$ : notice that the disk has performed a full revolution. Observe that the error decreases versus the mesh size. The error is plotted for the following variants of the method: (a) without mass correction, (b) with local mass correction (c) with local and global mass correction. The rate of convergence, i.e. the slope on the log-log graph, is respectively $0.30,0.46$ and 0.50 . For $N_{h}=400$, the mass lost is about $10 \%$ without any correction. It is improved to $4.9 \%$ the local correction and to $1.8 \%$ with both local and global corrections. Fig. 1.a shows the solution for $N_{h}=400$ with and without corrections: the corners become less rounded and the the disk is more conserved.

\section{References}

[1] Enright, D., Fedkin, R., Ferziger, J. et Mitchell, I., 2002. A hybrid partical level set method for improved interface capturing. J. Comput. Phys., 183, 83-116.

[2] Jiang, G.-S. et Peng, D., 2000. Weighted eno schemes for hamilton-jacobi equations. SIAM J. Sci. Comput., 21, $2126-2143$. 

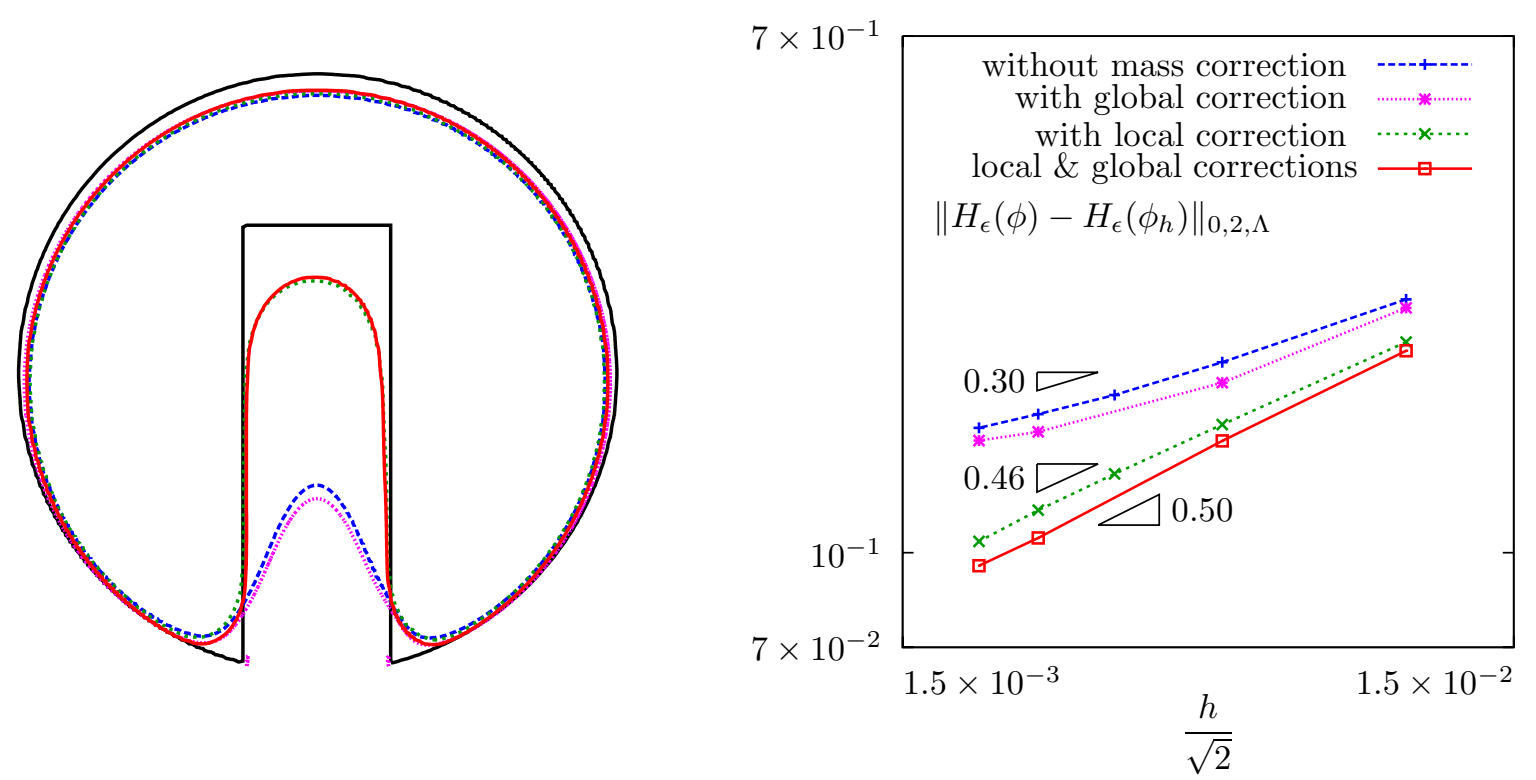

FIG. 1. The Zalesak disk: (a) initial position (black and dotted) and after one rotation: without correction (blue and dotted line), with global correction (magenta and dashed), with local correction (green and dashed), with local and global corrections (red and solid line) ; (b) convergence properties in $\log -\log$ scale.

Fig. 1. Le disque de Zalesak: (a) position initiale (trait noir et pointillé) et apès une rotation: sans correction (bleu et pointillé), avec correction globale (violet et pointillé), avec correction locale (vert et pointillé), avec corrections locale et globale (rouge et continu) ; (b) propriétés de convergence en échelle $\log$-log.

[3] Osher, S. et Fedkiw, R., 2003. The Level Set Method and Dynamic Implicit Surfaces. Springer-Verlag, New York.

[4] Pironneau, O., 1982. On the transport-diffusion algorithm and its applications to the Navier-Stokes equations. Numerische Mathematik, 38(3), 309-332.

[5] Saramito, P., Roquet, N. et Étienne, J., 2008. Rheolef: A finite element environment, i.e. some C++ classes and unix commands. http://www-lmc.imag.fr/lmc-edp/Pierre.Saramito/rheolef.

[6] Sethian, J., 1999. Level Set Methods and Fast Marching Methods. Cambridge University Press.

[7] Sussman, M. et Fatemi, E., 1998. An efficient, interface preserving level set re-distancing algorithm and its application to interfacial incompressible fluid flow. SIAM J. Sci. Comput, 20(4), 1165-1191.

[8] Zalesak, S., 1979. Fully multidimensional flux-corrected trasport algorithms for fluids. J. Comput. Phys., 31, 335-362. 